\title{
Marine chitinolytic enzymes, a biotechnological treasure hidden in the ocean?
}

Azadeh Beygmoradi, Ahmad Homaei, Roohullah Hemmati, Paloma Santos-Moriano, Daniel

Hormigo, Jesús Fernández-Lucas

\section{Abstract}

Chitinolytic enzymes are capable to catalyze the chitin hydrolysis. Due to their biomedical and biotechnological applications, nowadays chitinolytic enzymes have attracted worldwide attention. Chitinolytic enzymes have provided numerous useful materials in many different industries, such as food, pharmaceutical, cosmetic, or biomedical industry. Marine enzymes are commonly employed in industry because they display better operational properties than animal, plant, or bacterial homologs. In this mini-review, we want to describe marine chitinolytic enzymes as versatile enzymes in different biotechnological fields. In this regard, interesting comments about their biological role, reaction mechanism, production, functional characterization, immobilization, and biotechnological application are shown in this work.

Keywords:

Marine enzymes, Chitin hydrolysis, Biotechnological applications, Enzymatic production, Enzyme immobilization 